\title{
Core/Shell Magic-Sized Semiconductor Nanocrystals
}

\author{
Andrew B. Pun, Aniket S. Mule, Jacob T. Held, and David J. Norris* \\ Optical Materials Engineering Laboratory, \\ Department of Mechanical and Process Engineering, ETH Zurich, 8092 Zurich, Switzerland
}

\begin{abstract}
Magic-sized semiconductor nanocrystals (MSNCs) grow via discrete jumps between specific sizes. Despite their potential to offer atomically precise structures, their use has been limited by poor stability and trap-dominated photoluminescence. Recently, syntheses have been reported that produce CdSe MSNCs over a larger size series. We exploit such particles and demonstrate a method to grow shells on CdSe MSNC cores. Thin CdS shells lead to dramatic improvements in the emissive properties of the MSNCs, narrowing their fluorescence linewidths, enhancing photoluminescence quantum yields, and eliminating trap emission. While thicker CdS shells lead to decreased performance, $\mathrm{Cd}_{\mathrm{x}} \mathrm{Zn}_{1-\mathrm{x}} \mathrm{S}$ alloyed shells maintain efficient and narrow fluorescence lines. These alloyed core/shell crystallites also exhibit a tetrahedral shape, in agreement with a recent model for MSNC growth. Our results indicate that MSNCs can compete with other state-of-the-art semiconductor nanocrystals. Furthermore, these core/shell structures will allow further study of MSNCs and their potential for atomically precise growth.
\end{abstract}

KEYWORDS. core/shell nanocrystals, CdSe/CdS/ZnS, magic-sized clusters, discrete growth, atomically precise 
Semiconductor nanocrystals (NCs) exhibit optical properties that depend on their size and shape. ${ }^{1}$ Consequently, NC researchers have strived to develop syntheses that produce monodisperse (i.e. identical) NCs. ${ }^{2}$ These efforts have yielded high-quality quasi-spherical nanocrystals known as quantum dots (QDs), as well as a variety of other shapes, such as quasi-onedimensional nanorods, ${ }^{3}$ quasi-two-dimensional nanoplatelets, ${ }^{4}$ and tetrapods. ${ }^{5}$ However, despite these advances, the synthesis of monodisperse NCs with perfect uniformity is (perhaps unsurprisingly) still not possible. Thus, samples always exhibit inhomogeneous optical properties. For example, even for the best samples, single-NC spectroscopy reveals variations in fluorescence spectra between crystallites. ${ }^{6}$ These differences are at least in part due to distributions in size and shape. ${ }^{7}$

This leads to a fundamental question: how atomically precise can nanocrystals be ${ }^{8,9}$ One potential route to truly monodisperse NCs (at least in principle) is through magic-sized clusters (MSCs). ${ }^{10-12}$ While conventional QDs grow by continual addition of atoms, leading to a quasicontinuum of potential sizes, MSCs grow through discrete jumps such that only certain sizes are allowed..$^{13}$ Previous studies have indicated that MSCs are tetrahedral in shape, ${ }^{14,15}$ and we have recently shown that this can explain their discrete sizes. ${ }^{16}$ MSCs grow layer by layer, and the addition of a full layer on any one of the four exposed facets of the tetrahedron can yield the nextlarger tetrahedron in a series. This process involves a kinetic barrier, as a partially completed layer costs energy due to its extra dangling bonds. Upon completion of the layer, these extra dangling bonds disappear, causing a sudden decrease in energy. Thus, "magic" sizes appear due to a series of complete tetrahedra that represent local energy minima with kinetic barriers in between. This rationalizes why only complete tetrahedra are experimentally observed.

However, while the existence of MSCs has long been known, ${ }^{17,18}$ they have suffered from some disadvantages compared to other NCs, preventing their wider study and application. Notably, 
the photoluminescence (PL) of MSCs has been dominated by a very broad emission feature with a large Stokes shift from the lowest-energy absorption peak. ${ }^{19}$ Because this feature is reminiscent of "deep trap" emission in QDs, it has been presumed to originate from surface traps. ${ }^{20}$ Thus, careful tailoring of the MSC surface could lead to improved optical properties. ${ }^{21}$

Poor PL due to surface defects is not limited to MSCs, but is a common issue among semiconductor $\mathrm{NCs}^{22,23}$ One strategy to eliminate trap states is to coat the particle with a shell. ${ }^{24}$ The shell material serves to passivate dangling bonds of the optically active core and isolate it from the surrounding medium. ${ }^{25}$ The shell typically consists of a wide-band-gap semiconductor, which helps confine photogenerated charge carriers (electrons and holes) to the NC core. This approach has been applied to many NC shapes and structures, yielding particles with high photoluminescence quantum yields (PLQYs). ${ }^{26-29}$ In the case of CdSe NCs, common shells include other II-VI semiconductors with wider band gaps, such as CdS, ZnSe, ZnS, or their alloys. ${ }^{27,30-32}$

Clearly, the same approach could potentially be used to improve the poor PL of MSCs. One issue is that MSCs have been limited to very small sizes, which are typically hard to isolate and study. Fortunately, discrete growth is now possible to larger sizes. ${ }^{11,14,16}$ In particular, our group has recently introduced a synthesis that can produce up to 11 discrete species of CdSe crystallites. As their sizes extend well beyond the traditional cluster regime, we refer to the entire series as magic-sized nanocrystals (MSNCs). ${ }^{16}$ The larger particles can be easily isolated, providing discretely growing CdSe NCs that are stable under ambient conditions for months. ${ }^{16}$ They present an opportunity to grow shells on magic-sized particles.

Herein, we exploit this opportunity to synthesize core/shell CdSe MSNCs. We first isolate a specific MSNC to carefully control the size of our cores. We then deposit a shell to enhance the optical properties of these MSNCs. By growing thin layers of CdS on CdSe, we are able to eliminate the trap emission of the parent cores. This is accompanied by significant increases in the 
PLQY and narrowing of the linewidth of the band-edge fluorescence. However, growth of thicker CdS shells leads to sharp drops in the PLQY of our MSNCs. We find that the addition of a zinc precursor to our shelling reactions allows $\mathrm{CdSe} / \mathrm{Cd}_{\mathrm{x}} \mathrm{Zn}_{1-\mathrm{x}} \mathrm{S}$ MSNCs with thicker shells to be obtained. Furthermore, we show that the unique physical properties of MSNCs, as compared to conventional QDs, have a dramatic impact on the properties of the resulting core/shell NCs. The resulting bright, stable core/shell particles also enable study and application of MSNCs as an important nanomaterial class.

We introduce our process with zincblende CdSe MSNCs that have their lowest-energy absorption feature at $494 \mathrm{~nm}\left(\mathrm{MSNC}_{494}\right.$, Figure 1a). Slight modifications were made to our previously reported synthesis to obtain these cores. ${ }^{16}$ (See sections S1 and S2 in the Supporting Information for detailed descriptions of our materials and synthetic methods.) In particular, MSNC494 was synthesized in hexadecane instead of 1-octadecene (ODE) to prevent the formation of poly(ODE),${ }^{33}$ and additional washing steps were carried out. Our $\mathrm{MSNC}_{494}$ cores already exhibit relatively strong, band-edge fluorescence with a small Stokes shift, but trap emission is still visible (Figure 1b). The PLQY of these $\mathrm{MSNC}_{494}$ cores was measured up to $28 \%$, however this number fell to $18 \%$ when only the band-edge fluorescence was considered. (See section S3 in the Supporting Information for optical-characterization methods.)

We have previously observed that CdSe MSNCs can grow from one size to the next in the absence of Se precursors via a ripening process. ${ }^{16}$ Thus, to prevent core growth during shelling, which would potentially lead to a mixture of MSNC sizes, the shell should be added at a lower temperature than that used to obtain the core. We can also exploit the fact that progressively higher temperatures are required to grow a MSNC to the next-larger size. Thus, the enhanced thermal stability of our larger MSNCs allowed shelling reactions at relatively high temperatures, which is beneficial for obtaining good shells and high PLQYs. ${ }^{34}$ 
Specifically, we combined cadmium oleate and the $\mathrm{MSNC}_{494}$ cores in ODE and heated the mixture to $180{ }^{\circ} \mathrm{C}$. At this reduced temperature, formation of poly(ODE) is not expected. ${ }^{33} \mathrm{~A}$ sulfur source in ODE was then continuously added. At $180{ }^{\circ} \mathrm{C}$, the shelling reaction is sufficiently below the $240{ }^{\circ} \mathrm{C}$ at which $\mathrm{MSNC}_{494}$ was grown that core growth due to ripening is prevented. For the sulfur source, either sulfur or acetyl sulfide can be used, with negligible differences in the optical properties of the final core/shell MSNCs (Figure S1 in the Supporting Information). The only major difference between precursors is that the shell growth is faster with acetyl sulfide than with elemental sulfur (see section S2 in the Supporting Information). Upon completion, the reaction was cooled to room temperature and the product was isolated and cleaned via precipitation three times with methyl acetate followed by redispersion in hexanes, yielding $\mathrm{MSNC}_{494} / \mathrm{CdS}$ core/shell MSNCs.

The addition of the CdS shell shifts the absorption spectrum of the parent $\mathrm{MSNC}_{494}$ to longer wavelengths (Figure 1a). Because CdSe and CdS have conduction bands with similar band offsets, a photoexcited electron in a $\mathrm{CdSe} / \mathrm{CdS}$ core/shell $\mathrm{NC}$ can delocalize to the shell, explaining this red shift. ${ }^{35}$ Indeed, it has been widely demonstrated that thicker CdS shells on CdSe NCs induce larger red shifts of the lowest-energy absorption feature relative to the CdSe core itself. ${ }^{36,37} \mathrm{We}$ observed a continuous red shift in the absorption of $\mathrm{MSNC}_{494} / \mathrm{CdS} \mathrm{NCs}$ with shell growth (Figure $\mathrm{S} 2$ in the Supporting Information), in contrast to the discrete jumps seen for the MSNC core. This is indicative of continuous shell growth.

Upon shelling, the fluorescence of $\mathrm{MSNC}_{494}$ dramatically improved. The low-energy trap emission observed in $\mathrm{MSNC}_{494}$ was completely eliminated by the addition of the shell, with $\mathrm{MSNC}_{494} / \mathrm{CdS}$ only exhibiting band-edge fluorescence (Figure 1b). The PLQY of $\mathrm{MSNC}_{494} / \mathrm{CdS}$ was also increased, up to $78 \%$. Finally, the fluorescence linewidth of $\mathrm{MSNC}_{494} \mathrm{CdS}$ narrowed to $116 \mathrm{meV}$, compared to $140 \mathrm{meV}$ for the original $\mathrm{MSNC}_{494}$ cores. 
A similar procedure was applied to smaller MSNCs, which exhibit a lowest-energy absorption feature at $434 \mathrm{~nm}\left(\mathrm{MSNC}_{434}\right.$, Figure 1c). Prior to adding a shell, the PL of $\mathrm{MSNC}_{434}$ also showed a band-edge feature, but emission from trap states was more pronounced (see Figure 1d) than in spectra for $\mathrm{MSNC}_{494}$. The total PLQY of $\mathrm{MSNC}_{434}$ was measured up to $38 \%$, but the band-edge contribution was only up to $12 \%$. Because the synthesis of the smaller $\mathrm{MSNC}_{434}$ was at $180{ }^{\circ} \mathrm{C}$ (see section S2 in the Supporting Information), shells were added at $150{ }^{\circ} \mathrm{C}$ to prevent core growth due to ripening. As with $\mathrm{MSNC}_{494} / \mathrm{CdS}$, a continuous red shift of the absorption features was seen during shell growth for $\mathrm{MSNC}_{434} / \mathrm{CdS}$ (Figure $\mathrm{S} 3$ in the Supporting Information). The trap emission that dominated the PL spectrum of the $\mathrm{MSNC}_{434}$ cores was almost completely eliminated in $\mathrm{MSNC}_{434} / \mathrm{CdS}$ (Figure 1d). The band-edge-only PLQY increased to up to 55\%, with a narrowing of the fluorescence linewidth from $161 \mathrm{meV}$ in the original $\mathrm{MSNC}_{434}$ cores to $141 \mathrm{meV}$.

We analyzed these core/shell MSNCs via transmission electron microscopy (TEM) and scanning TEM (STEM) and measured the projected area of our particles (see section S4 in the Supporting Information). While our previous work suggested that such MSNCs are truncated tetrahedra, ${ }^{16}$ we assume below that the MSNC cores and core/shell particles are quasi-spherical for ease of comparison. This allows us to convert the measured projected areas into effective diameters (see section S5 of the Supporting Information). Prior to shelling, $\mathrm{MSNC}_{494}$ had an average effective diameter of $2.67 \pm 0.22 \mathrm{~nm}$, which grew to $3.13 \pm 0.19 \mathrm{~nm}$ in the completed $\mathrm{MSNC}_{494} / \mathrm{CdS}$ (Figure 2a,b). Prior work has shown that one monolayer (ML) of CdS grown completely around a zincblende CdSe nanoplatelet core (i.e., on both sides) leads to a total thickness increase of $0.61 \mathrm{~nm} .{ }^{38}$ Thus, our $\mathrm{MSNC}_{494} / \mathrm{CdS}$ corresponds to the growth of $\sim 0.75 \mathrm{MLs}(0.46 \mathrm{~nm})$ of $\mathrm{CdS}$ around the $\mathrm{MSNC}_{494} \mathrm{CdSe}$ core. In the case of $\mathrm{MSNC}_{434}$, we measured an initial average effective diameter of $2.13 \pm 0.29 \mathrm{~nm}$, which increased to $2.52 \pm 0.21 \mathrm{~nm}$ for $\mathrm{MSNC}_{434} / \mathrm{CdS}$ (Figure $2 \mathrm{c}, \mathrm{d}$ ). This suggests a growth of 0.64 MLs $(0.39 \mathrm{~nm})$ of CdS. We note that we did not observe triangular 
projections in the STEM images, which would be a clear indication of tetrahedral-shaped particles. However, this is not surprising given the small particle sizes. Indeed, our earlier work on $\mathrm{MSNC}_{494}$ suggested faceted particles, but they were too small for a precise shape determination. ${ }^{16}$ Here, the addition of the sub-ML-thick shells would potentially further smoothen any surface facets.

The shell thicknesses extracted from electron microscopy can be further corroborated by experiments using colloidal atomic layer deposition (c-ALD) $.^{39} \mathrm{c}-\mathrm{ALD}$ proceeds via self-limiting half reactions of ionic precursors adding to a surface. ${ }^{40}$ For example, in the case of cadmium-rich surfaces of CdSe, a layer of sulfur can be deposited, followed by a layer of cadmium to yield $1 \mathrm{ML}$ of added CdS. This process, which is typically carried out at room temperature, has been shown to be efficient, ${ }^{38-40}$ adding $1 \mathrm{ML}$ of CdS with each full cycle (one layer of $\mathrm{S}$ and one layer of Cd). Multiple cycles can then yield thicker shells.

Consequently, c-ALD has been suggested as an alternative approach to core/shell MSNCs. ${ }^{9}$ However, we observed much poorer optical properties in our core/shell MSNCs grown via c-ALD when compared to those grown with our high-temperature synthesis. For example, the PLQY of $\mathrm{MSNC}_{494}$ with $1 \mathrm{ML}$ of $\mathrm{CdS}$ grown via c-ALD was only up to $7 \%$. Nevertheless, we can use cALD to further characterize our high-temperature shell growth. The lowest-energy absorption feature of our $\mathrm{MSNC}_{494} / \mathrm{CdS}$ is at $514 \mathrm{~nm}$, close to the $518 \mathrm{~nm}$ peak position for $\mathrm{MSNC}_{494}$ with 1 ML CdS added via c-ALD (Figure S4 in the Supporting Information). For MSNC434, our hightemperature shell growth yielded an absorption feature at $458 \mathrm{~nm}$, compared to $\sim 478 \mathrm{~nm}$ for $1 \mathrm{ML}$ of CdS added via c-ALD (Figure S5 in the Supporting Information). These results are consistent with our conclusion above that the MSNC/CdS samples in Figure 1 have a CdS shell just under 1 ML thick.

It has been observed that thicker CdS shells on a CdSe core often lead to higher PLQYs compared to thin shells. ${ }^{41,42}$ Therefore, we attempted to grow thicker shells on $\mathrm{MSNC}_{494}$ by 
modifying the amount of cadmium and sulfur precursors used, as well as by varying the reaction times (see section S2 in the Supporting Information). Absorption and PL spectra were taken at various time points as the shell was added (Figure 3). The absorption peaks of the resulting core/shell crystallites (which we refer to as $\mathrm{MSNC}_{494} / \mathrm{CdS}+$ ) are shifted to even longer wavelengths than those seen for our thin-shelled $\mathrm{MSNC}_{494} / \mathrm{CdS}$, consistent with the addition of more CdS. The $\mathrm{PL}$ of $\mathrm{MSNC}_{494} / \mathrm{CdS}+$ also remains sharp, with trap emission completely absent (Figure 3b, inset). However, the PL intensity drops rapidly between 60 and $120 \mathrm{~min}$. The final washed species has a PLQY only up to $10 \%$, much lower than the thin-shelled $\mathrm{MSNC}_{494} / \mathrm{CdS}$ above. When one monolayer of CdS was grown on $\mathrm{MSNC}_{494}$ via c-ALD, the PL peak was observed at $553 \mathrm{~nm}$ (grey dashed line, Figure 3b). The drop in PL intensity that we observe when growing CdS on CdSe $\mathrm{MSNC}_{494}$ occurs as we approach one monolayer of CdS. A similar and even more dramatic effect is seen when a thicker shell is grown on $\mathrm{MSNC}_{434}$ (Figure $\mathrm{S6}$ in the Supporting Information). The PLQY of this species ( $\mathrm{MSNC}_{434} / \mathrm{CdS}+$ ) is only up to $3 \%$.

To investigate why thicker CdS shells lead to poor optical properties, we performed a TEM size analysis. It revealed that the $\mathrm{MSNC}_{494} / \mathrm{CdS}+$ have an average effective diameter of $3.73 \pm 0.54 \mathrm{~nm}$. This is an increase of $1.05 \mathrm{~nm}$ from the $\mathrm{MSNC}_{494}$ core and corresponds to $\sim 1.7 \mathrm{ML}$ of CdS. The $\mathrm{MSNC}_{494} / \mathrm{CdS}+$ particles do not appear tetrahedral (Figure S7 in the Supporting Information). If they were tetrahedral, we should observe triangular projections at this size, as we did previously for MSNCs with an effective diameter of $3.27 \mathrm{~nm} .{ }^{16}$ In addition, the STEM images show particles with enhanced aggregation, as compared to $\mathrm{MSNC}_{494} / \mathrm{CdS}$ in Figure $2 \mathrm{~b}$. In the case of $\mathrm{MSNC}_{434} / \mathrm{CdS}+$, we again see particles with no clear triangular projections, with an effective diameter of $3.26 \pm 0.84 \mathrm{~nm}$, corresponding to the growth of $1.8 \mathrm{MLs}$ of CdS. Here many long worm-like particle aggregates are visible in STEM images (Figure S8 in the Supporting Information). 
These data suggest that growth of CdS shells above $1 \mathrm{ML}$ on our CdSe MSNCs leads to a steep drop in PL intensity. We postulate that the observed shape and aggregation of these particles results from uneven shell growth to relieve strain. Due to the lattice mismatch between CdSe and CdS $(\sim 4 \%)$, the edges and corners of the tetrahedral MSNC cores exhibit increasing strain as the shell growth proceeds. We believe this enhanced strain leads to the poor optical properties in CdSe MSNCs with thicker CdS shells.

Alloying is a common strategy to reduce interfacial strain in core/shell NCs. ${ }^{43-45}$ In particular $\mathrm{Cd}_{\mathrm{x}} \mathrm{Zn}_{1-\mathrm{x}} \mathrm{S}$ grown on $\mathrm{CdSe}$ has led to core/shell particles with excellent optoelectronic properties. ${ }^{27,46} \mathrm{ZnS}$ has a wider band gap than $\mathrm{CdS}$, leading to better passivation of the CdSe core. However, $\mathrm{ZnS}$ has a larger lattice mismatch with $\mathrm{CdSe}(\sim 12 \%){ }^{30-32}$ The alloyed shell leads to reduced interfacial strain as compared to a pure $\mathrm{ZnS}$ shell, while still maintaining the benefits of the wider band gap of $\mathrm{ZnS}$.

To grow a $\mathrm{Cd}_{\mathrm{x}} \mathrm{Zn}_{1-\mathrm{x}} \mathrm{S}$ shell on our $\mathrm{MSNC}_{494}$, we modified our earlier $\mathrm{CdS}$ procedure. Specifically, we added a zinc precursor (zinc oleate) and oleylamine along with the cadmium precursor (cadmium oleate); elemental sulfur was used as the sulfur source (see section S2 in the Supporting Information). These precursors were previously exploited to obtain $\mathrm{Cd}_{\mathrm{x}} \mathrm{Zn}_{1-\mathrm{x}} \mathrm{S}$ shells on QDs and nanoplatelets. ${ }^{29,47,48}$ As with the growth of CdS on MSNCs above, we observed a gradual red shift of the absorption features as the reaction proceeds, indicative of continuous shell growth (Figure S9 in the Supporting Information). After the addition of the shell, the isolated crystallites exhibited a lowest-energy absorption peak at $534 \mathrm{~nm}$, with strong PL (up to 80\% PLQY) without trap emission (Figure $4 \mathrm{a}$ ). This $\sim 40 \mathrm{~nm}$ red shift in the lowest-energy absorption feature is larger (smaller) than that expected for a shell of pure $\mathrm{ZnS}(\mathrm{CdS}) .{ }^{39,49,50}$ Thus, this observation is consistent with the addition of an alloyed shell. 
TEM images of these $\mathrm{MSNC}_{494} / \mathrm{Cd}_{\mathrm{x}} \mathrm{Zn}_{1-\mathrm{x}} \mathrm{S}$ confirmed that we had grown thick shells on our initial $\mathrm{MSNC}_{494}$ cores (Figure $4 \mathrm{~b}$ ). The average effective diameter of $\mathrm{MSNC}_{494} / \mathrm{Cd}_{\mathrm{x}} \mathrm{Zn}_{1-\mathrm{x}} \mathrm{S}$ was $5.16 \pm 0.47 \mathrm{~nm}$, an increase of $2.49 \mathrm{~nm}$ from the $\mathrm{MSNC}_{494}$ core. Most strikingly, the MSNC $494 / \mathrm{Cd}_{\mathrm{x}} \mathrm{Zn}_{1-\mathrm{x}} \mathrm{S}$ crystallites clearly exhibit triangular projections in STEM, as we would expect for tetrahedral-shaped particles. Assuming that they are indeed tetrahedral, we can determine their effective edge length. Converting from the projected area (section S5 in the Supporting Information) gives us an effective edge length of $6.95 \pm 0.63 \mathrm{~nm}$. To determine whether this tetrahedral shape is a result of growing on a tetrahedral core or due to the shelling conditions, we synthesized zincblende QDs, ${ }^{51}$ which are known to be quasi-spherical. In particular, we grew QDs with a lowest-energy absorption feature at $494 \mathrm{~nm}\left(\mathrm{QD}_{494}\right.$, Figure S10 in the Supporting Information), matching the peak position of $\mathrm{MSNC}_{494}$. We then added $\mathrm{Cd}_{\mathrm{x}} \mathrm{Zn}_{1-\mathrm{x}} \mathrm{S}$ shells on these QD 494 with the same method as with $\mathrm{MSNC}_{494} / \mathrm{Cd}_{\mathrm{x}} \mathrm{Zn}_{1-\mathrm{x}} \mathrm{S}$. The resulting $\mathrm{QD} 494 / \mathrm{Cd}_{\mathrm{x}} \mathrm{Zn}_{1-\mathrm{x}} \mathrm{S}$ shows similar optical properties to $\mathrm{MSNC}_{494} / \mathrm{Cd}_{\mathrm{x}} \mathrm{Zn}_{1-\mathrm{x}} \mathrm{S}$, with longer-wavelength absorbance and PL without trap emission (Figure S11 in the Supporting Information). However, TEM images of $\mathrm{QD}_{494} / \mathrm{Cd}_{\mathrm{x}} \mathrm{Zn}_{1-\mathrm{x}} \mathrm{S}$ reveal that these particles have irregular shapes (Figure S12 in the Supporting Information) without discernable triangular projections. Thus, we conclude that the triangular projections observed for $\mathrm{MSNC}_{494} / \mathrm{Cd}_{\mathrm{x}} \mathrm{Zn}_{1-\mathrm{x}} \mathrm{S}$ are due to the shape of the underlying $\mathrm{MSNC}_{494}$ core. We note that while previous data has suggested that small CdSe MSNCs are tetrahedral, ${ }^{14,15}$ it had been unclear if this holds for all MSNCs. ${ }^{9,13}$ Thus, in addition to enhanced optical properties, our core/shell MSNCs can give us details about structure of the underlying MSNC core.

In conclusion, we have demonstrated the synthesis of core/shell crystallites grown from CdSe MSNCs. Thin CdS shells dramatically improve the optical properties of MSNCs, leading to an elimination of low-energy emission from trap states, a decrease in the fluorescence linewidth, and large increases in PLQY. Analysis of these particles via TEM shows these thin CdS shells are 
below 1 ML thick. Thicker CdS shells can also be grown, but this leads to diminished PLQY. TEM of particles with thicker shells reveals quasi-spherical shapes that tend to aggregate. To obtain MSNCs with thick shells with high PLQY, we grew $\mathrm{Cd}_{\mathrm{x}} \mathrm{Zn}_{1-\mathrm{x}} \mathrm{S}$ shells. Unlike our MSNCs with thick CdS shells, our MSNC ${ }_{494} / \mathrm{Cd}_{\mathrm{x}} \mathrm{Zn}_{1-\mathrm{x}} \mathrm{S}$ species is clearly tetrahedral. These results demonstrate a route to overcome the poor stability and poor optical properties that have plagued MSCs. Furthermore, these core/shell structures will enable the fundamental structural and optoelectronic properties of MSNCs to be studied, expanding our understanding of this special class of nanomaterial.

\section{ASSOCIATED CONTENT}

\section{Supporting Information}

The Supporting Information is available free of charge on the ACS Publications website at DOI: $10.1021 / \mathrm{xxxxxxx}$

Experimental materials and methods, including details of the synthesis, characterization, and size analysis of the MSNC cores, core/shell MSNCs, and core/shell QDs.

\section{AUTHOR INFORMATION}

\section{Corresponding Author}

*Email: dnorris@ethz.ch.

\section{ORCID}

Andrew B. Pun: 0000-0002-3052-912X

Aniket S. Mule: 0000-0001-8387-080X

Jacob T. Held: 0000-0003-3864-4314

David J. Norris: 0000-0002-3765-0678 


\section{Funding}

This work was supported by the Swiss National Science Foundation (SNSF) under Award No. 200021-188593. A.B.P. acknowledges funding from the ETH Zurich Postdoctoral Fellowship (Marie Curie Actions).

\section{ACKNOWLEDGMENTS}

We thank M. Aellen, S. Mazzotti, and H. Rojo for technical assistance and helpful discussions. This work benefitted from facilities at the Scientific Center for Optical and Electronic Microscopy (ScopeM) at ETH Zurich.

\section{REFERENCES}

(1) Efros, Al. L.; Brus, L. E. Nanocrystal Quantum Dots: From Discovery to Modern Development. ACS Nano 2021, 15, 6192-6210.

(2) Murray, C. B.; Norris, D. J.; Bawendi, M. G. Synthesis and Characterization of Nearly Monodisperse CdE (E = Sulfur, Selenium, Tellurium) Semiconductor Nanocrystallites. $J$. Am. Chem. Soc. 1993, 115, 8706-8715.

(3) Peng, X.; Manna, L.; Yang, W.; Wickham, J.; Scher, E.; Kadavanich, A.; Alivisatos, A. P. Shape Control of CdSe Nanocrystals. Nature 2000, 404, 59-61.

(4) Ithurria, S.; Dubertret, B. Quasi 2D Colloidal CdSe Platelets with Thicknesses Controlled at the Atomic Level. J. Am. Chem. Soc. 2008, 130, 16504-16505.

(5) Manna, L.; Milliron, D. J.; Meisel, A.; Scher, E. C.; Alivisatos, A. P. Controlled Growth of Tetrapod-Branched Inorganic Nanocrystals. Nat. Mater. 2003, 2, 382-385.

(6) Chen, O.; Zhao, J.; Chauhan, V. P.; Cui, J.; Wong, C.; Harris, D. K.; Wei, H.; Han, H.-S.; Fukumura, D.; Jain, R. K.; Bawendi, M. G. Compact High-Quality CdSe-CdS Core-Shell Nanocrystals with Narrow Emission Linewidths and Suppressed Blinking. Nat. Mater. 2013, 12, 445-451.

(7) Kim, B. H.; Heo, J.; Kim, S.; Reboul, C. F.; Chun, H.; Kang, D.; Bae, H.; Hyun, H.; Lim, J.; Lee, H.; Han, B.; Hyeon, T.; Alivisatos, A. P.; Ercius, P.; Elmlund, H.; Park, J. Critical Differences in 3D Atomic Structure of Individual Ligand-Protected Nanocrystals in Solution. Science 2020, 368, 60-67.

(8) Hens, Z.; De Roo, J. Atomically Precise Nanocrystals. J. Am. Chem. Soc. 2020, 142, $15627-15637$.

(9) Palencia, C.; Yu, K.; Boldt, K. The Future of Colloidal Semiconductor Magic-Size Clusters. ACS Nano 2020, 14, 1227-1235.

(10) Kudera, S.; Zanella, M.; Giannini, C.; Rizzo, A.; Li, Y.; Gigli, G.; Cingolani, R.; Ciccarella, G.; Spahl, W.; Parak, W. J.; Manna, L. Sequential Growth of Magic-Size CdSe Nanocrystals. Adv. Mater. 2007, 19, 548-552.

(11) Cossairt, B. M.; Owen, J. S. CdSe Clusters: At the Interface of Small Molecules and 
Quantum Dots. Chem. Mater. 2011, 23, 3114-3119.

(12) Wang, F.; Wang, Y.; Liu, Y.-H.; Morrison, P. J.; Loomis, R. A.; Buhro, W. E. TwoDimensional Semiconductor Nanocrystals: Properties, Templated Formation, and MagicSize Nanocluster Intermediates. Acc. Chem. Res. 2015, 48, 13-21.

(13) Pun, A. B.; Mazzotti, S.; Mule, A. S.; Norris, D. J. Understanding Discrete Growth in Semiconductor Nanocrystals: Nanoplatelets and Magic-Sized Clusters. Acc. Chem. Res. 2021, 54, 1545-1554.

(14) Beecher, A. N.; Yang, X.; Palmer, J. H.; LaGrassa, A. L.; Juhas, P.; Billinge, S. J. L.; Owen, J. S. Atomic Structures and Gram Scale Synthesis of Three Tetrahedral Quantum Dots. J. Am. Chem. Soc. 2014, 136, 10645-10653.

(15) Yang, X.; Masadeh, A. S.; McBride, J. R.; Božin, E. S.; Rosenthal, S. J.; Billinge, S. J. L. Confirmation of Disordered Structure of Ultrasmall CdSe Nanoparticles from X-Ray Atomic Pair Distribution Function Analysis. Phys. Chem. Chem. Phys. 2013, 15, 84808486.

(16) Mule, A. S.; Mazzotti, S.; Rossinelli, A. A.; Aellen, M.; Prins, T.; van der Bock, J. C.; Solari, S. F.; Glauser, Y. M.; Kumar, P. V; Riedinger, A.; Norris, D. J. Unraveling the Growth Mechanism of Magic-Sized Semiconductor Nanocrystals. J. Am. Chem. Soc. 2021, 143, 2037-2048.

(17) Dance, I. G.; Choy, A.; Scudder, M. L. Syntheses, Properties, and Molecular and Crystal Structures of $\left(\mathrm{Me}_{4} \mathrm{~N}\right)_{4}\left[\mathrm{E}_{4} \mathrm{M}_{10}(\mathrm{SPh})_{16}\right](\mathrm{E}=$ Sulfur or Selenium; $\mathrm{M}=$ Zinc or Cadmium): Molecular Supertetrahedral Fragments of the Cubic Metal Chalcogenide Lattice. J. Am. Chem. Soc. 1984, 106, 6285-6295.

(18) Herron, N.; Suna, A.; Wang, Y. Synthesis of $\approx 10 \AA$ Thiophenolate-Capped CdS Clusters. Observation of a Sharp Absorption Peak. J. Chem. Soc., Dalton Trans. 1992, 2329-2335.

(19) VanWie, T.; Wysocki, E.; McBride, J. R.; Rosenthal, S. J. Bright Cool White Emission from Ultrasmall CdSe Quantum Dots. Chem. Mater. 2019, 31, 8558-8562.

(20) Bowers II, M. J.; McBride, J. R.; Garrett, M. D.; Sammons, J. A.; Dukes III, A. D.; Schreuder, M. A.; Watt, T. L.; Lupini, A. R.; Pennycook, S. J.; Rosenthal, S. J. Structure and Ultrafast Dynamics of White-Light-Emitting CdSe Nanocrystals. J. Am. Chem. Soc. 2009, 131, 5730-5731.

(21) Cossairt, B. M.; Juhas, P.; Billinge, S. J. L.; Owen, J. S. Tuning the Surface Structure and Optical Properties of CdSe Clusters Using Coordination Chemistry. J. Phys. Chem. Lett. 2011, 2, 3075-3080.

(22) Kim, W.; Lim, S. J.; Jung, S.; Shin, S. K. Binary Amine-Phosphine Passivation of Surface Traps on CdSe Nanocrystals. J. Phys. Chem. C 2010, 114, 1539-1546.

(23) Anderson, N. C.; Hendricks, M. P.; Choi, J. J.; Owen, J. S. Ligand Exchange and the Stoichiometry of Metal Chalcogenide Nanocrystals: Spectroscopic Observation of Facile Metal-Carboxylate Displacement and Binding. J. Am. Chem. Soc. 2013, 135, 1853618548.

(24) Reiss, P.; Protière, M.; Li, L. Core/Shell Semiconductor Nanocrystals. Small 2009, 5, 154 168.

(25) Gómez-Campos, F. M.; Califano, M. Hole Surface Trapping in CdSe Nanocrystals: Dynamics, Rate Fluctuations, and Implications for Blinking. Nano Lett. 2012, 12, 45084517.

(26) Carbone, L.; Nobile, C.; De Giorgi, M.; Sala, F. Della; Morello, G.; Pompa, P.; Hytch, M.; Snoeck, E.; Fiore, A.; Franchini, I. R.; Nadasan, M.; Silvestre, A. F.; Chiodo, L.; Kudera, S.; Cingolani, R.; Krahne, R.; Manna, L. Synthesis and Micrometer-Scale Assembly of 
Colloidal CdSe/CdS Nanorods Prepared by a Seeded Growth Approach. Nano Lett. 2007, 7, 2942-2950.

(27) Boldt, K.; Kirkwood, N.; Beane, G. A.; Mulvaney, P. Synthesis of Highly Luminescent and Photo-Stable, Graded Shell CdSe/Cd $\mathrm{Zn}_{1-\mathrm{x}} \mathrm{S}$ Nanoparticles by In Situ Alloying. Chem. Mater. 2013, 25, 4731-4738.

(28) Jeong, B. G.; Park, Y.-S.; Chang, J. H.; Cho, I.; Kim, J. K.; Kim, H.; Char, K.; Cho, J.; Klimov, V. I.; Park, P.; Lee, D. C.; Bae, W. K. Colloidal Spherical Quantum Wells with Near-Unity Photoluminescence Quantum Yield and Suppressed Blinking. ACS Nano 2016, 10, 9297-9305.

(29) Rossinelli, A. A.; Rojo, H.; Mule, A. S.; Aellen, M.; Cocina, A.; De Leo, E.; Schäublin, R.; Norris, D. J. Compositional Grading for Efficient and Narrowband Emission in CdSeBased Core/Shell Nanoplatelets. Chem. Mater. 2019, 31, 9567-9578.

(30) Hines, M. A.; Guyot-Sionnest, P. Synthesis and Characterization of Strongly Luminescing ZnS-Capped CdSe Nanocrystals. J. Phys. Chem. 1996, 100, 468-471.

(31) Peng, X.; Schlamp, M. C.; Kadavanich, A. V; Alivisatos, A. P. Epitaxial Growth of Highly Luminescent $\mathrm{CdSe} / \mathrm{CdS}$ Core/Shell Nanocrystals with Photostability and Electronic Accessibility. J. Am. Chem. Soc. 1997, 119, 7019-7029.

(32) Dabbousi, B. O.; Rodriguez-Viejo, J.; Mikulec, F. V; Heine, J. R.; Mattoussi, H.; Ober, R.; Jensen, K. F.; Bawendi, M. G. (CdSe)ZnS Core-Shell Quantum Dots: Synthesis and Characterization of a Size Series of Highly Luminescent Nanocrystallites. J. Phys. Chem. B 1997, 101, 9463-9475.

(33) Dhaene, E.; Billet, J.; Bennett, E.; Driessche, I. Van; Roo, J. De. The Trouble with 1Octadecene; Polymerization During Nanocrystal Synthesis. Nano Lett. 2019, 19, $7411-$ 7417.

(34) Ji, B.; Koley, S.; Slobodkin, I.; Remennik, S.; Banin, U. ZnSe/ZnS Core/Shell Quantum Dots with Superior Optical Properties through Thermodynamic Shell Growth. Nano Lett. 2020, 20, 2387-2395.

(35) Rossinelli, A. A.; Riedinger, A.; Marqués-Gallego, P.; Knüsel, P. N.; Antolinez, F. V; Norris, D. J. High-Temperature Growth of Thick-Shell CdSe/CdS Core/Shell Nanoplatelets. Chem. Commun. 2017, 53, 9938-9941.

(36) van Embden, J.; Jasieniak, J.; Mulvaney, P. Mapping the Optical Properties of CdSe/CdS Heterostructure Nanocrystals: The Effects of Core Size and Shell Thickness. J. Am. Chem. Soc. 2009, 131, 14299-14309.

(37) Lin, W.; Niu, Y.; Meng, R.; Huang, L.; Cao, H.; Zhang, Z.; Qin, H.; Peng, X. ShellThickness Dependent Optical Properties of CdSe/CdS Core/Shell Nanocrystals Coated with Thiol Ligands. Nano Res. 2016, 9, 260-271.

(38) Hazarika, A.; Fedin, I.; Hong, L.; Guo, J.; Srivastava, V.; Cho, W.; Coropceanu, I.; Portner, J.; Diroll, B. T.; Philbin, J. P.; Rabani, E.; Klie, R.; Talapin, D. V. Colloidal Atomic Layer Deposition with Stationary Reactant Phases Enables Precise Synthesis of "Digital" II-VI Nano-Heterostructures with Exquisite Control of Confinement and Strain. J. Am. Chem. Soc. 2019, 141, 13487-13496.

(39) Ithurria, S.; Talapin, D. V. Colloidal Atomic Layer Deposition (c-ALD) Using SelfLimiting Reactions at Nanocrystal Surface Coupled to Phase Transfer between Polar and Nonpolar Media. J. Am. Chem. Soc. 2012, 134, 18585-18590.

(40) Slejko, E. A.; Sayevich, V.; Cai, B.; Gaponik, N.; Lughi, V.; Lesnyak, V.; Eychmüller, A. Precise Engineering of Nanocrystal Shells via Colloidal Atomic Layer Deposition. Chem. Mater. 2017, 29, 8111-8118. 
(41) Nan, W.; Niu, Y.; Qin, H.; Cui, F.; Yang, Y.; Lai, R.; Lin, W.; Peng, X. Crystal Structure Control of Zinc-Blende CdSe/CdS Core/Shell Nanocrystals: Synthesis and StructureDependent Optical Properties. J. Am. Chem. Soc. 2012, 134, 19685-19693.

(42) Meinardi, F.; Colombo, A.; Velizhanin, K. A.; Simonutti, R.; Lorenzon, M.; Beverina, L.; Viswanatha, R.; Klimov, V. I.; Brovelli, S. Large-Area Luminescent Solar Concentrators Based on 'Stokes-Shift-Engineered' Nanocrystals in a Mass-Polymerized PMMA Matrix. Nat. Photonics 2014, 8, 392-399.

(43) Grumbach, N.; Capek, R. K.; Tilchin, E.; Rubin-Brusilovski, A.; Yang, J.; Ein-Eli, Y.; Lifshitz, E. Comprehensive Route to the Formation of Alloy Interface in Core/Shell Colloidal Quantum Dots. J. Phys. Chem. C 2015, 119, 12749-12756.

(44) Lim, J.; Park, Y.-S.; Klimov, V. I. Optical Gain in Colloidal Quantum Dots Achieved with Direct-Current Electrical Pumping. Nat. Mater. 2018, 17, 42-49.

(45) Altintas, Y.; Gungor, K.; Gao, Y.; Sak, M.; Quliyeva, U.; Bappi, G.; Mutlugun, E.; Sargent, E. H.; Demir, H. V. Giant Alloyed Hot Injection Shells Enable Ultralow Optical Gain Threshold in Colloidal Quantum Wells. ACS Nano 2019, 13, 10662-10670.

(46) Bae, W. K.; Padilha, L. A.; Park, Y.-S.; McDaniel, H.; Robel, I.; Pietryga, J. M.; Klimov, V. I. Controlled Alloying of the Core-Shell Interface in CdSe/CdS Quantum Dots for Suppression of Auger Recombination. ACS Nano 2013, 7, 3411-3419.

(47) Manna, L.; Scher, E. C.; Li, L.-S.; Alivisatos, A. P. Epitaxial Growth and Photochemical Annealing of Graded CdS/ZnS Shells on Colloidal CdSe Nanorods. J. Am. Chem. Soc. 2002, 124, 7136-7145.

(48) Xie, R.; Kolb, U.; Li, J.; Basché, T.; Mews, A. Synthesis and Characterization of Highly Luminescent $\mathrm{CdSe}-$ Core $\mathrm{CdS} / \mathrm{Zn}_{0.5} \mathrm{Cd}_{0.5} \mathrm{~S} / \mathrm{ZnS}$ Multishell Nanocrystals. J. Am. Chem. Soc. 2005, 127, 7480-7488.

(49) Hao, J.; Liu, H.; Miao, J.; Lu, R.; Zhou, Z.; Zhao, B.; Xie, B.; Cheng, J.; Wang, K.; Delville, M.-H. A Facile Route to Synthesize CdSe/ZnS Thick-Shell Quantum Dots with Precisely Controlled Green Emission Properties: Towards QDs Based LED Applications. Sci. Rep. 2019, 9, 12048.

(50) Greytak, A. B.; Allen, P. M.; Liu, W.; Zhao, J.; Young, E. R.; Popović, Z.; Walker, B. J.; Nocera, D. G.; Bawendi, M. G. Alternating Layer Addition Approach to CdSe/CdS Core/Shell Quantum Dots with near-Unity Quantum Yield and High on-Time Fractions. Chem. Sci. 2012, 3, 2028-2034.

(51) Shen, H.; Wang, H.; Tang, Z.; Niu, J. Z.; Lou, S.; Du, Z.; Li, L. S. High Quality Synthesis of Monodisperse Zinc-Blende CdSe and CdSe/ZnS Nanocrystals with a Phosphine-Free Method. CrystEngComm 2009, 11, 1733-1738. 

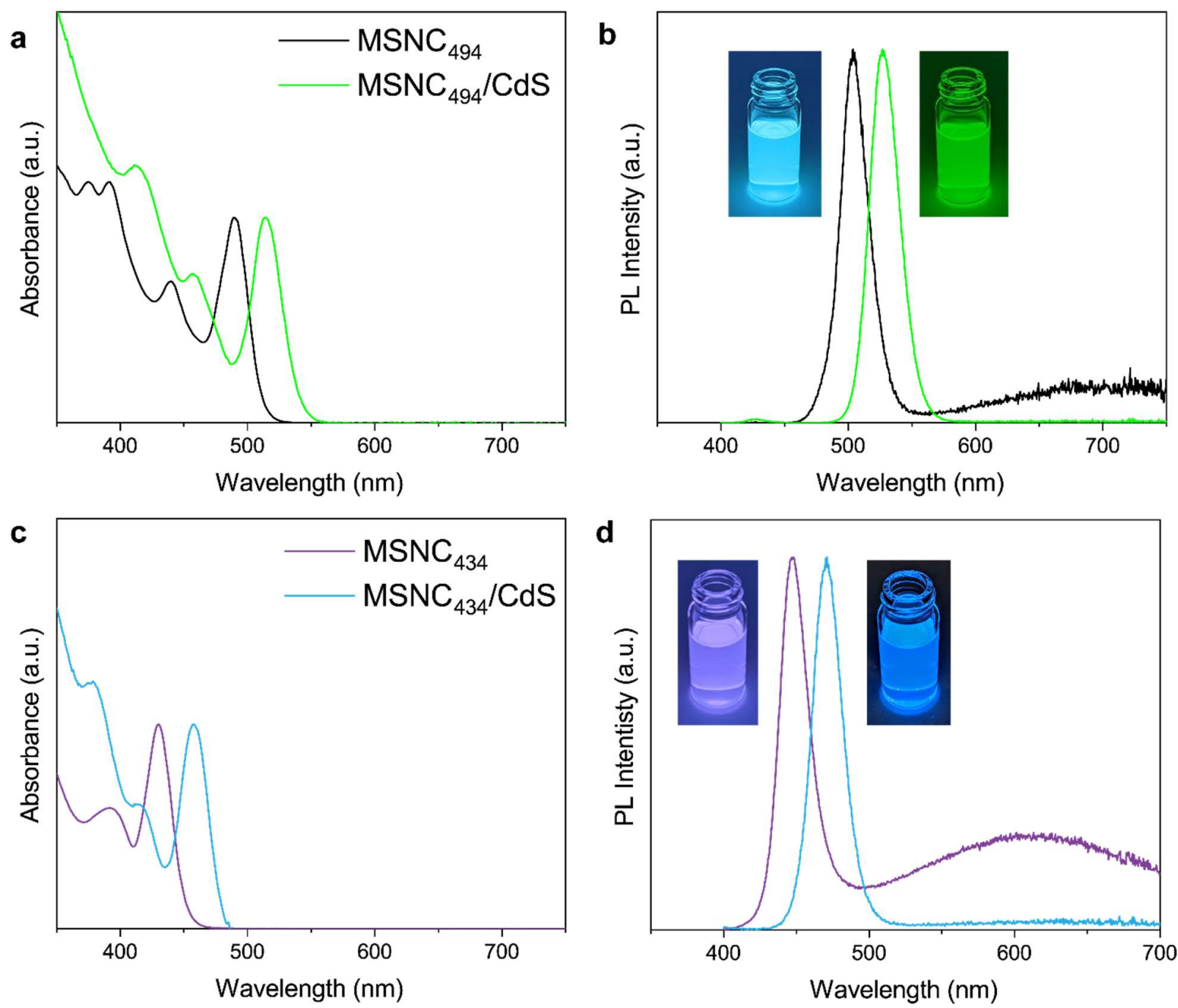

Figure 1. Room-temperature optical properties of magic-sized nanocrystal (MSNC) cores and core/shell crystallites. (a),(b) Absorption and photoluminescence (PL) spectra for MSNC 494 with and without a thin CdS shell. For PL, the samples were excited at $380 \mathrm{~nm}$. (c),(d) Same as in (a),(b) except for $\mathrm{MSNC}_{434}$ with and without a thin CdS shell. For PL, the samples were excited at $380 \mathrm{~nm}$. The insets in (b),(d) show hexane dispersions of the MSNCs under $365 \mathrm{~nm}$ illumination. For the shell growth, acetyl sulfide was used as the sulfur precursor. 
a

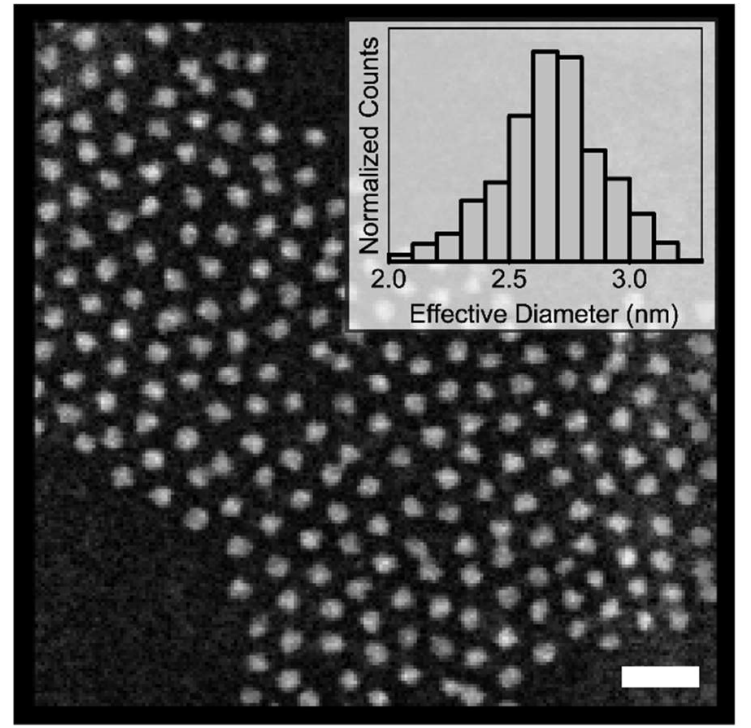

C

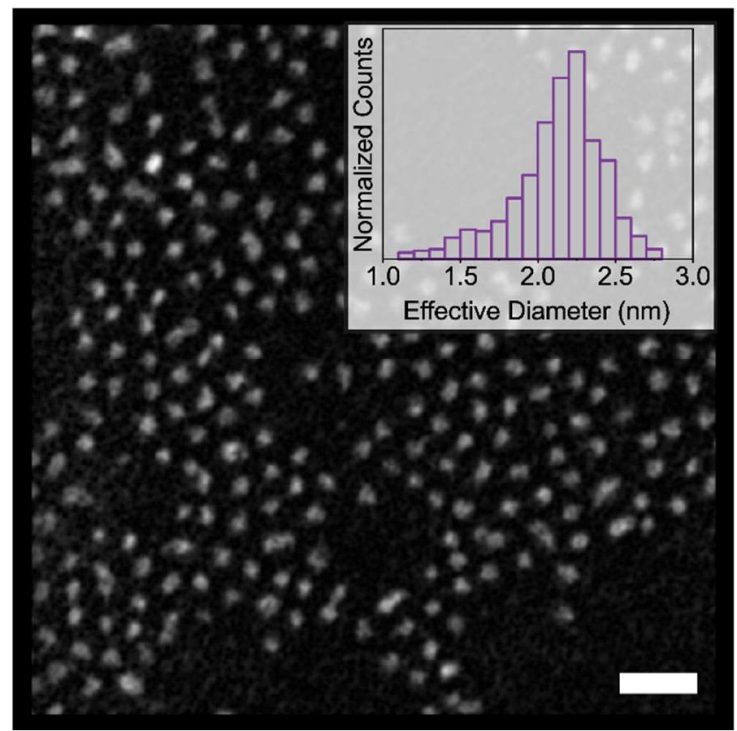

b

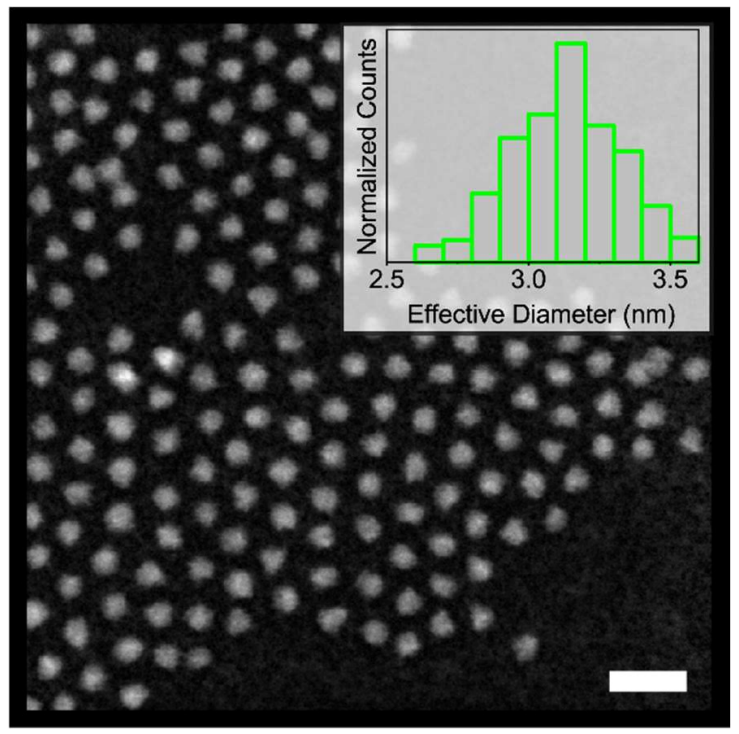

d

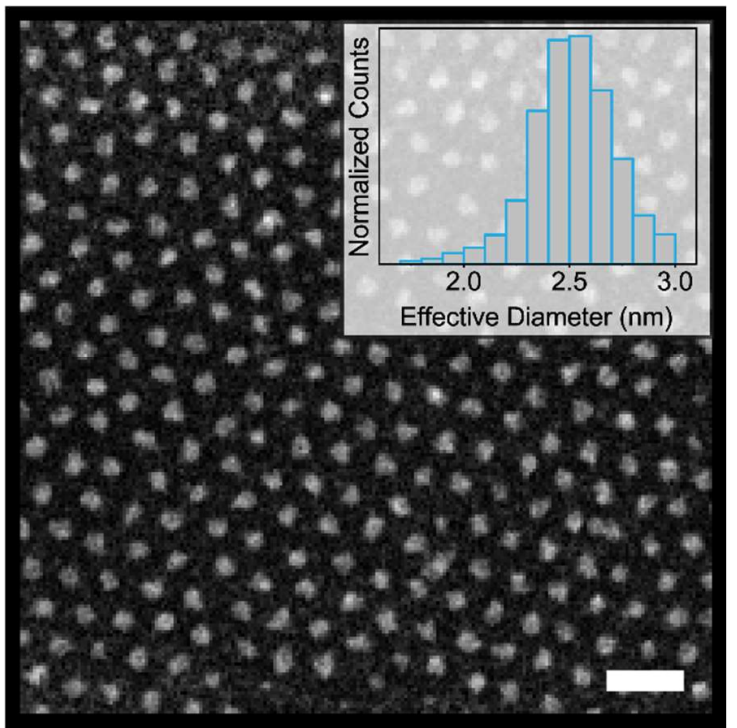

Figure 2. Electron-microscopy characterization of MSNC cores and core/shell crystallites. Representative high-angle annular dark field scanning transmission electron microscope (HAADFSTEM) images for (a) $\mathrm{MSNC}_{494}$, (b) $\mathrm{MSNC}_{494} / \mathrm{CdS}$, (c) $\mathrm{MSNC}_{434}$, and (d) $\mathrm{MSNC}_{434} / \mathrm{CdS}$. The insets show the distribution of effective diameters for (a) 869, (b) 677, (c) 670, and (d) 1398 particles. The average effective diameters of these particles are (a) $2.67 \pm 0.22 \mathrm{~nm}$, (b) $3.13 \pm 0.19 \mathrm{~nm}$, (c) $2.13 \pm 0.29 \mathrm{~nm}$, and (d) $2.52 \pm 0.21 \mathrm{~nm}$. The increase in size corresponds to a $\sim 0.75 \mathrm{ML}$ thick $\mathrm{CdS}$ shell for $\mathrm{MSNC}_{494} / \mathrm{CdS}$ and $\sim 0.64 \mathrm{ML}$ for $\mathrm{MSNC}_{434} / \mathrm{CdS}$. Scale bars are $10 \mathrm{~nm}$. We note that while strategies to minimize electron-beam damage in STEM images were followed (see section S4 in the Supporting Information), the images are still potentially affected. 

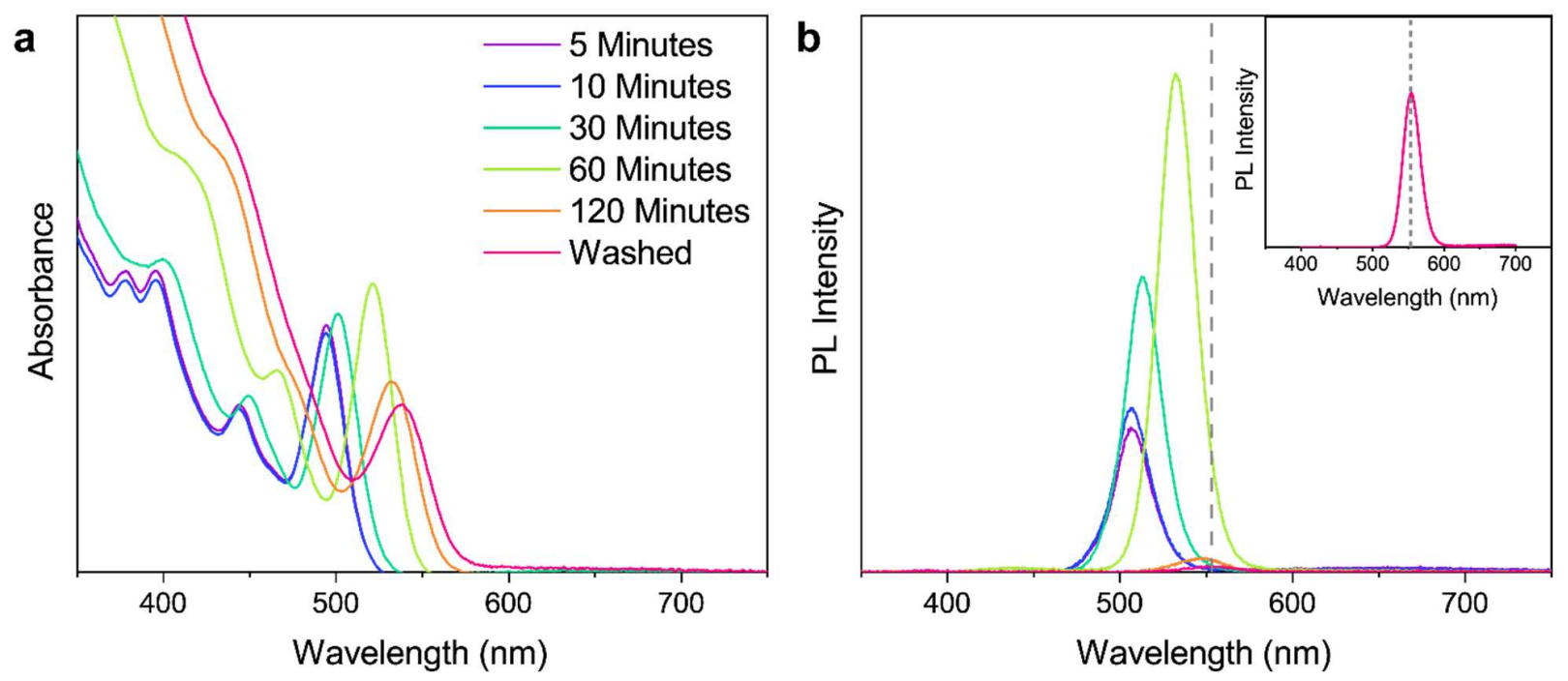

Figure 3. Optical properties of $\mathrm{MSNC}_{494}$ crystallites during addition of thick CdS shells. Roomtemperature (a) absorption and (b) photoluminescence (PL) spectra at various time points during the growth of $\mathrm{MSNC}_{494} / \mathrm{CdS}+$. For PL, the samples were excited at $380 \mathrm{~nm}$. The grey dashed vertical line in panel $b$ corresponds to the PL peak of $\mathrm{MSNC}_{494}$ with one monolayer of $\mathrm{CdS}$ added via colloidal atomic-layer deposition (c-ALD). The inset in (b) shows the PL spectrum of the final washed $\mathrm{MSNC}_{494} / \mathrm{CdS}+$. 

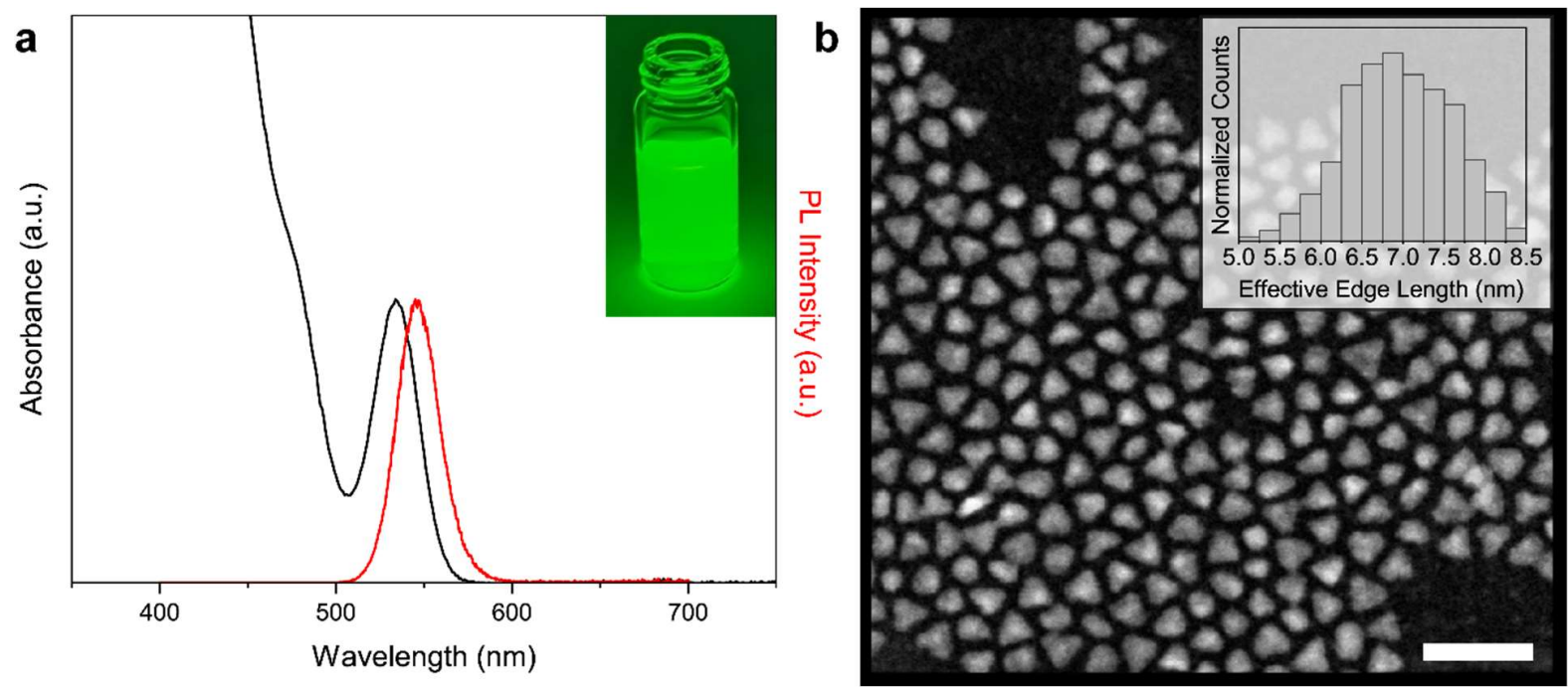

Figure 4. Optical and structural characterization of $\mathrm{MSNC}_{494} / \mathrm{Cd}_{\mathrm{x}} \mathrm{Zn}_{1-\mathrm{x}} \mathrm{S}$ crystallites. (a) Roomtemperature absorption (black) and photoluminescence (PL, red) of our $\mathrm{MSNC}_{494} / \mathrm{Cd}_{\mathrm{x}} \mathrm{Zn}_{1-\mathrm{x}} \mathrm{S}$. For PL, the sample was illuminated at $380 \mathrm{~nm}$. The inset shows a hexane dispersion of $\mathrm{MSNC}_{494} / \mathrm{Cd}_{\mathrm{x}} \mathrm{Zn}_{1-\mathrm{x}} \mathrm{S}$ under $365 \mathrm{~nm}$ illumination. (b) Representative STEM image of $\mathrm{MSNC}_{494} / \mathrm{Cd}_{\mathrm{x}} \mathrm{Zn}_{1-\mathrm{x}} \mathrm{S}$ with an extracted effective edge length of $6.95 \pm 0.63 \mathrm{~nm}$ (assuming equilateral triangles), corresponding to a $\sim 4 \mathrm{ML}$ thick shell grown on the $\mathrm{MSNC}_{494}$ cores. The inset shows the distribution of effective edge lengths of 603 particles assuming equilateral triangles. The scale bar is $20 \mathrm{~nm}$. We note that while strategies to minimize electron-beam damage in STEM images were followed (see section S4 in the Supporting Information), the images are still potentially affected. 
TABLE OF CONTENTS GRAPHIC

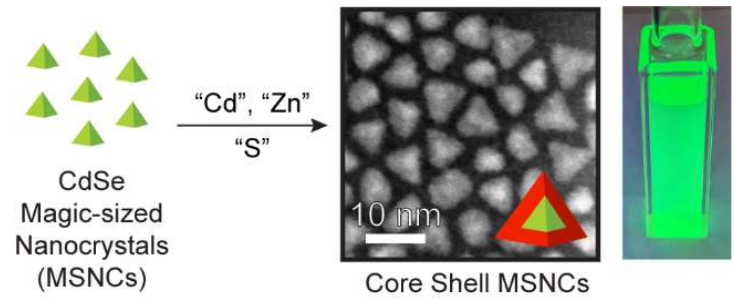

